\title{
Dinámica del NDVI en distintas fases del fenómeno ENSO en la Reserva de Biósfera Laguna Blanca (Catamarca, Argentina)
}

\author{
Alejandro E. Maggi ${ }^{\bowtie}$; Karen D. Ponieman; Nicolás G. Castro \& Miguel Di \\ FERDinANDO
}

Universidad de Buenos Aires. Facultad de Agronomía. Cátedra de Manejo y Conservación de Suelos.

\begin{abstract}
Resumen. El Niño-Oscilación del Sur (ENSO, por sus siglas en inglés) es un fenómeno oceánico-climático que muestra dos fases contrastantes y una fase intermedia o neutra que afectan a diferentes regiones del mundo. En algunas ecorregiones del Noroeste Argentino (NOA), dichas fases provocarían años con precipitaciones menores o mayores que el promedio histórico, correspondientes a El Niño y La Niña, respectivamente. Estas diferencias en la disponibilidad de agua causan cambios en la cobertura vegetal y en la degradación de la tierra. Los cambios producidos en el régimen hídrico por causas naturales o antrópicas afectan la productividad de los diferentes ecosistemas y se pueden inferir a través de las diferencias en los índices espectrales, como el Índice de Vegetación de Diferencia Normalizada (NDVI). Los objetivos de esta investigación fueron caracterizar la dinámica de la cobertura vegetal estimada mediante el NDVI y estudiar las relaciones temporales entre el ENSO, la precipitación y el NDVI en las comunidades más conspicuas de la Puna en la Reserva de Biósfera Laguna Blanca. Se obtuvieron datos de NDVI del sensor MODIS (Moderate Resolution Imaging Spectroradiometer) a partir de un mapa publicado de comunidades vegetales de la reserva; la precipitación, a partir del GPCC (Global Precipitation Climatology Centre) y el Índice Oceánico El Niño (ONI, por sus siglas en inglés), de la National Oceanic and Atmospheric Administration (NOAA). La máxima diferencia de este último índice entre las distintas fases del ENSO se alcanzó entre septiembre y febrero. Como consecuencia del régimen monzónico, las precipitaciones alcanzaron el máximo un trimestre después; las más altas fueron en eventos La Niña. El máximo NDVI también mostró un retraso de uno a dos trimestres respecto al ONI dependiendo la comunidad analizada. Se encontraron indicios que prueban la conexión entre el NDVI, las precipitaciones y las fases del ENSO en la Puna catamarqueña. En años extremos La Niña y El Niño, tanto en estepas de la Puna árida y semiárida se manifiestan diferencias significativas en el NDVI. Los resultados sugieren que el monitoreo del ONI permitiría anticipar la aplicación de estrategias adecuadas para el control de la desertificación.
\end{abstract}

[Palabras clave: comunidades vegetales, precipitación, estepas, Puna, desertificación, series de NDVI MODIS]

Aвstract. NDVI dynamics in different phases of the ENSO phenomenon in the Laguna Blanca Biosphere Reserve (Catamarca, Argentina). El Niño-Southern Oscillation (ENSO) is an oceanic-climatic phenomenon which shows two contrasting phases and an intermediate or neutral phase that affect different regions of the world. In some ecoregions of northwestern Argentina (NOA), these phases would induce years with lower or higher rainfall than the historical average, corresponding to El Niño and La Niña, respectively. These differences in water availability cause changes in vegetation cover and land degradation, as well. Changes in the water regime due to natural or anthropic causes, which affect the productivity of different ecosystems, can be inferred through differences in spectral indices, such as NDVI (Normalized Difference Vegetation Index). The main objectives of this research were to characterize the dynamics of the vegetation cover estimated by mean of NDVI and study the temporal relationships among ENSO, rainfall and NDVI in the most conspicuous communities of the Puna in the Laguna Blanca Biosphere Reserve. NDVI data from the MODIS sensor (Moderate Resolution Imaging Spectroradiometer) were obtained from a published map of plant communities in the reserve; precipitation, from the GPCC (Global Precipitation Climatology Center), and the El Niño Oceanic Index (ONI), from the National Oceanic and Atmospheric Administration (NOAA). The highest difference of this last index among the different phases of the ENSO was reached between September and February. As a consequence of the monsoon regime, rainfall reached its peak a quarter later, being the highest in La Niña events. The maximum NDVI also showed a delay of one to two quarters with respect to ONI depending on the community considered. Results suggest that there is a connection among NDVI, rainfall and ENSO phases in Catamarca's Puna. In extreme years La Niña and El Niño, both in steppes of the arid and semi-arid Puna, significant differences in NDVI values were observed. The results suggest that ONI monitoring would allow anticipating the application of appropriate strategies for desertification control.

[Keywords: plant communities, rainfall, steppes, Puna, desertification, series of NDVI MODIS]

Editor asociado: Germán Baldi \raggi@agro.uba.ar
Recibido: 5 de Agosto de 2019

Aceptado: 19 de Diciembre de 2019 


\section{INTRODUCCIÓN}

Alrededor del 50\% del territorio continental argentino presenta precipitaciones menores a $400 \mathrm{~mm}$ anuales, lo cual representa una limitación para la agricultura en secano. Estas tierras se destinan a la ganadería (i.e., bovinos, ovinos, caprinos y camélidos) sobre pastizales naturales. El Noroeste Argentino (NOA) no escapa a esta realidad, a la que se suma que las lluvias escasas pueden alcanzar una intensidad elevada en los períodos estivales y provocar procesos de erosión hídrica en grandes extensiones; en particular, en aquellas áreas de relieve pronunciado, en las que la débil cohesión de los materiales superficiales del suelo y la poca protección vegetal favorecen dichos procesos. A estos factores geográficos se suman factores socio-económicos. El área no es homogénea en cuanto a las economías familiares y al manejo del ambiente. Los pobladores de bajo nivel económico suelen eliminar los arbustos, que constituyen su única fuente de combustible. Además, sus animales sobrepastorean los pastizales naturales, lo que genera áreas extensas de suelo desnudo. Estos "peladales" son focos de erosión eólica e hídrica (i.e., formación de surcos y cárcavas) cuando predominan los vientos erosivos y en épocas de lluvias, respectivamente (Movia and Navone 1993).

El control del conjunto de fenómenos descriptos evitaría el desarrollo de un complejo proceso de desertificación (UN 1994). Esto requeriría de un manejo sustentable del sistema ganadero que evite el pastoreo sin control y conserve la diversidad vegetal. En la región de la Puna, el porcentaje de cobertura vegetal es uno de los indicadores que más explica el proceso de desertificación. Tal proceso está asociado a que el $61.8 \%$ de las explotaciones tienen una carga superior a la receptividad de la zona y el $86 \%$ usa como combustible leña de plantas conocidas como tola (Parastrephia sp., Fabiana sp. y Baccharis sp.) y de otras especies como Azorella compacta (Yareta) y Polylepsis sp. (Queñoa) (Maccagno 2004). La productividad primaria neta aérea (PPNA) de las distintas comunidades vegetales de la Puna oscila entre 3900 kg MS.ha-1.año ${ }^{-1}$ en las estepas graminosas y $472 \mathrm{~kg} \mathrm{MS}^{-h^{-1}}$ año $^{-1}$ en las estepas arbustivas (Baldassini et al. 2012). Sin embargo, no existen suficientes estudios que aborden cómo la variabilidad climática afecta la productividad de las diferentes comunidades vegetales. Esta información básica es necesaria para diseñar planes estratégicos de manejo de estos sistemas. El panorama se torna más complejo cuando se considera el fenómeno El Niño-Oscilación del Sur (ENOS o ENSO, El Niño-Southern Oscillation, por sus siglas en inglés) y su probable intensificación en un escenario de calentamiento global (Maggi et al. 2010; Maggi and Di Ferdinando 2012; Worcel and Maggi 2016).

El fenómeno ENSO implica grandes intercambios de calor entre el océano y la atmósfera que afectan a la temperatura media global de la Tierra y crean situaciones extremas en el ciclo hidrológico, como pueden ser lluvias torrenciales y sequías en diferentes partes del mundo. El proceso ENSO muestra dos fases extremas y una fase intermedia o neutra que afectan diferentes regiones del mundo, con intensidades que varían de muy débil a muy fuerte en cada una de ellas. La fase cálida del ciclo (El Niño) se caracteriza por un debilitamiento a gran escala de los vientos alisios y un calentamiento de la superficie del mar en el Océano Pacífico ecuatorial central y del este. Por el contrario, la fase fría (La Niña) es consecuencia de un enfriamiento atípico del Océano Pacífico este. La Niña intensifica el funcionamiento de la celda de Walker al reforzar los vientos alisios, acumular aguas cálidas al oeste del Pacífico y fortalecer la divergencia ecuatorial y la surgencia frente a las costas de Ecuador, de Perú y del norte de Chile (Maturana et al. 1997).

Otro fenómeno a mayor escala temporal planetaria a tener en cuenta es la Oscilación Decadal del Pacífico (PDO, Pacific Decadal Oscillation, por sus siglas en inglés) (Mantua and Hare 2002). El cambio de fase de la PDO se produjo antes de 1980. Tanto en la región semiárida de Colorado, Estados Unidos (Chen et al. 2017), como en la Argentina fue identificado para la región semiárida central (Minetti and Vargas 1997; Agosta and Compagnucci 2008) y para la Puna argentina (Barrera and Maggi 2018). Tales procesos de escala global causados por interacciones entre la atmósfera y el océano, como el ENSO y la PDO, afectan la temperatura y la precipitación en los Andes (Vuille and Bradley 2000; Garreaud and Aceituno 2007). Sin embargo, no existen estudios contundentes que demuestren la conexión entre el ENSO y la ocurrencia de las precipitaciones en la Puna seca del noroeste argentino.

Además, como ya fuera demostrado en varios informes del IPCC (Intergovernmental Panel on Climate Change) existe un incremento global de la temperatura, que coincide con indicadores 
climáticos como la retirada general de los glaciares y el aumento en la temperatura de la superficie del mar o cantidad de días secos consecutivos (Barros 2004; Barros and Camilloni 2016; First 2019). Los modelos de los escenarios climáticos para fin de siglo muestran que la tendencia general para las regiones secas será convertirse en tierras más secas (IPCC 2014). Estudios realizados en los Andes Tropicales revelan que el calentamiento global podría influir sobre el ENSO al potenciar los efectos de sus fases, en especial El Niño, que se vería favorecido por el aumento de temperaturas (Tsonis et al. 2003). Debido a la ubicación subtropical y a sus altitudes, superiores a 4000 m s. n. m., se espera que los Andes Centrales se vuelvan más cálidos y secos, con precipitaciones entre un 10 y un 30\% menores en el siglo XXI, lo que afectará poblaciones, ecosistemas y glaciares del altiplano en Sudamérica (Minvielle and Garreaud 2011). Thibeault et al. (2011), modelando las precipitaciones para el siglo XXI en el altiplano boliviano, llegan a conclusiones similares y predicen también una disminución de la frecuencia de precipitaciones en diciembre, enero y febrero a partir del año 2020, junto con una reducción del volumen de lluvias a fines de primavera y principios de verano. Estos autores, además, predicen un incremento de la evapotranspiración, lo que favorece que el suelo se seque y se endurezca entre episodios de lluvia, incrementando así el escurrimiento superficial y la erosión hídrica.

Sin embargo, en el sector sur de los Andes Centrales ocupado por la Puna seca, son muy pocos los estudios de las tendencias del clima para predecir el cambio climático en futuros escenarios. En particular, la información sobre la influencia del ENSO es limitada en el NOA. Como consecuencia de la ocurrencia de este fenómeno, por lo general se producen años con menores y mayores precipitaciones al promedio histórico en la región de la Puna, correspondientes a las fases El Niño y La Niña, respectivamente. A pesar de ello, los registros pluviométricos muestran una gran variabilidad espacial y temporal (Trauth et al. 2000). Esa variabilidad dificulta la posibilidad de detectar patrones de respuesta a las distintas fases del fenómeno ENSO.

La comprensión de tal variación espacial y temporal de la lluvia y la temperatura es muy importante a la hora de realizar proyecciones climáticas. De acuerdo con estudios previos en otras partes de Sudamérica, las temperaturas en esta región de los Andes se ven afectadas por el ENSO, lo que resultará en años calurosos, especialmente en los veranos durante los eventos de El Niño (Vuille and Bradley 2000; Seiler et al. 2013). Por su parte, la precipitación disminuyó durante la temporada de lluvias en las últimas tres décadas en la mayoría de los análisis de las estaciones que se encuentran en el suroeste de la Puna chilena, aunque las tendencias no fueron significativas (Vuille et al. 2003; Bennett et al. 2016). Resultados similares se han encontrado en la Puna boliviana (Seiler et al. 2013) y en la Puna jujeña de la Argentina (Barrera and Maggi 2018).

Los cambios que se produjeron en el régimen hídrico, tanto por causas naturales como antrópicas, afectan de manera directa los servicios y la productividad de los diversos ecosistemas. Dichos efectos sobre la productividad se pueden inferir a través del Índice Verde Normalizado (IVN o NDVI, Normalized Difference Vegetation Index, por sus siglas en inglés) (Paruelo and Lauenroth 1998). El NDVI es un buen estimador de la cantidad de radiación fotosintéticamente activa interceptada por el follaje verde y, por lo tanto, de la PPNA (Sellers et al. 1992). El mismo, responde positivamente a los incrementos tanto en el área que ocupa una planta (PAI, Plant Area Index) como en el Índice de Área Foliar. Por esa razón, el NDVI resulta también un buen estimador de la cobertura vegetal (Carlson and Ripley 1997; Gaitán et al. 2013). En ese sentido se desarrollaron diversas ecuaciones que permiten relacionar la cobertura vegetal de distintos tipos fisonómicos de la Puna de Jujuy con el NDVI obtenido de las imágenes MODIS (del inglés, Moderate Resolution Imaging Spectroradiometer) y las oscilaciones del clima (Maggi and Ponieman 2018; Ponieman et al. 2018).

Por otra parte, las características multitemporales de los índices obtenidos de imágenes satelitales los convirtieron en una herramienta útil para el estudio de fenómenos ambientales dinámicos y complejos, como la desertificación. Estas características permiten un monitoreo deforma rápida y económica para que la integración de las imágenes satelitales en Sistemas de Información Geográfica (SIG) sean de gran utilidad en la toma de decisiones y la formulación de políticas de conservación en los ámbitos públicos y privados (Espoz-Alsina et al. 2002). El satélite Terra, lanzado el 18 de diciembre de 1999, pertenece a una serie de 
satélites del Sistema de Observación Terrestre (EOS), un programa de largo plazo dedicado a comprender cómo los cambios naturales e inducidos por el hombre afectan al ambiente. Uno de los sensores a bordo de dicho satélite es el MODIS, que cubre la superficie terrestre cada 2 días y adquiere datos en 36 bandas espectrales a una resolución espacial de 250, 500 y 1000 m (Gao et al. 2003).

A partir de este análisis surge la necesidad de comprender las relaciones entre la cobertura de suelo de las distintas comunidades vegetales representativas de la Puna seca con las precipitaciones de las distintas fases del fenómeno ENSO, cuya variabilidad impacta en la degradación de las tierras. El objetivo principal de este trabajo es caracterizar la dinámica de la cobertura vegetal estimada mediante el NDVI en la Reserva de Biósfera Laguna Blanca (Catamarca, Argentina). Como objetivo específico se plantea estudiar las relaciones temporales entre el NDVI, la precipitación y el ENSO. Considerando estos objetivos, se propone como hipótesis que el NDVI permite discriminar las distintas comunidades de la Reserva de Biósfera Laguna Blanca (Catamarca, Argentina). En la Puna seca argentina, la fase El Niño del fenómeno ENSO está relacionada con mayores valores del ONI y con menores precipitaciones en el período monzónico (i.e., estación lluviosa durante octubre a abril en el NOA). Por su parte, para la fase La Niña es posible determinar la cantidad de meses entre la ocurrencia de la expresión de un índice climático ONI, que permite establecer una fase del ENSO, y la máxima respuesta del NDVI durante la ocurrencia del período monzónico en la Reserva.

\section{Materiales y MÉtodos}

Para obtener los datos estimados de precipitaciones mensuales de la Reserva Laguna Blanca se utilizó la grilla de $0.5^{\circ}$ de latitud por $0.5^{\circ}$ de longitud del GPCC (Global Precipitation Climatology Centre) debido a que en la región de estudio no se encuentran suficientes estaciones meteorológicas. Dado que el punto central de la reserva se ubica en el límite de cuatro pixeles, se calculó el promedio de los mismos para cada mes. Los datos fueron obtenidos del GPCC Land surface Full Data Monthly Product Version 2018 $0.5^{\circ}$. Se realizó una comparación mes a mes del promedio de los valores entre las diferentes fases del ENSO.
El Índice Oceánico de El Niño (ONI, Oceanic Niño Index, por sus siglas en inglés) para cada mes se calcula como la media de 3 meses consecutivos (i.e., el mes en cuestión y el mes anterior y posterior) de las anomalías de la temperatura superficial del mar medidas por el sensor ERSST.v3 en la región 3.4 de El Niño $\left(5^{\circ} \mathrm{N}-5^{\circ} \mathrm{S} ; 120^{\circ} \mathrm{E}-170^{\circ} \mathrm{O}\right)$, tomando como referencia el período de 1971 - 2000. Se consideran episodios cálidos y fríos cuando las anomalías superan el umbral de $\pm 0.5^{\circ} \mathrm{C}$. Cuando dicho umbral es cubierto durante un mínimo de 5 meses consecutivos se definen como episodios del Niño (+) o de la Niña (-). Para definir a qué fase ENSO correspondió cada año se analizaron los datos de cada período monzónico (octubre-abril) de acuerdo con la metodología desarrollada por Barrera y Maggi (2018), que considera una ponderación del índice mensual de ONI, registrado por la National Oceanic and Atmospheric Administration (NOAA), por el porcentaje de la precipitación media climática mensual del período octubreabril. En el período 2000/2016 se determinaron seis eventos La Niña, cuatro El Niño y seis Neutro. Para cada fase, en cada mes se calculó el promedio y el error estándar de los valores de precipitación estimados.

El índice de vegetación NDVI fue obtenido a partir de imágenes MOD13Q1 del sensor MODIS TERRA (resolución temporal de 16 días). Para la serie 2000/2016 a analizar no se incluyeron los meses de enero y febrero del año 2000, en los que las imágenes aún no estaban disponibles para los usuarios. Para caracterizar el tipo fisonómico de cada pixel de la reserva se utilizó el mapa temático de ambientes elaborado por Borgnia et al. (2006) (Figura 1). El mapa de comunidades vegetales identifica tres clases de estepas con características fisionómicas diferentes (i.e., arbustivas, graminosas y mixtas), y dentro de cada clase se diferencian comunidades según la especie dominante por lo que finalmente se obtuvieron 8 comunidades. Entre las comunidades arbustivas se destacan Fabiana densa J. Rémy (tolilla alta), Fabiana friesii Dammer y Fabiana punensis S. C. Arroyo (tolilla baja) y Acantholippia salsoloides Griseb.; entre las gramíneas se encuentran las comunidades de Stipa frigida Phil. y Stipa vaginata Phil., Festuca argentinensis (St.-Yves) Türpe y Panicum chloroleucum Griseb.; mientras que en la estepa mixta se encuentran las comunidades de Stipa sp. y Panicum chloroleucum Griseb. Se compararon los promedios de NDVI de cada comunidad, siguiendola metodología utilizada 


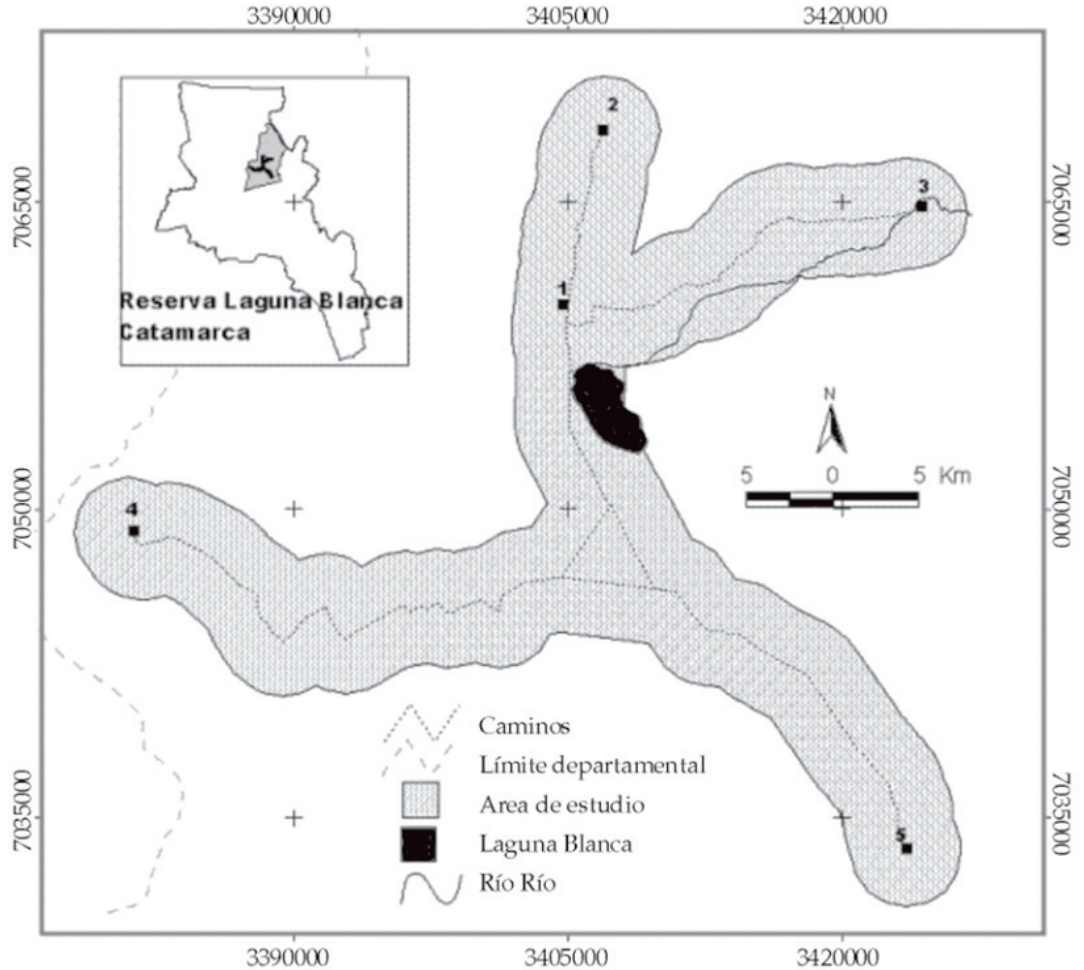

Figura 1. Área de estudio en la Reserva de Biósfera Laguna Blanca, perteneciente a Parques Nacionales. Los números indican lo siguiente: 1=Pueblo de Laguna Blanca; 2=Pueblo de Corral Blanco; 3=Puesto del Río; 4=Puesto Guzmán (Pasto Ventura); 5=Quebrada de Randolfo. Fuente: Borgnia et al. 2006.

Figure 1. Study area in the Laguna Blanca Biosphere Reserve, which belongs to National Parks. Numbers indicate the following: $1=$ Town of Laguna Blanca; $2=$ Town of Corral Blanco; $3=$ Site of the cattle breeder near the river: 4=Site of the cattle breeder Guzman (Pasto Ventura); 5=Quebrada de Randolfo. Source: Borgnia et al. 2006. por Maggi y Di Ferdinando (2012), en la que los autores usaron el mapa de comunidades vegetales elaborado por Ruthsatz y Movia (1975) y establecieron áreas de interés o muestreo equivalentes a 100 píxeles MODIS para cada comunidad en el período 2000-2011. Se aplicó un ANOVA (Tukey $\alpha=0.05$ ), realizado con el Software INFOSTAT para demostrar la existencia de diferencias en los valores medios de NDVI entre las comunidades mapeadas, independientemente de la época del año y del fenómeno ENSO.

Luego, se seleccionaron dos comunidades, de Panicum chloroleucum y de Fabiana densa, representativas de las estepas graminosas y arbustivas, respectivamente, debido a que ambas ocupan elevada extensión dentro de cada tipo fisonómico y muestran elevados y bajos valores de NDVI respectivamente. Las mismas se utilizaron para caracterizar la dinámica de la cobertura vegetal en las distintas fases del ENSO. Por medio de un análisis similar al de Aralova et al. (2016), para cada mes considerado se calculó la integral de tres meses para las precipitaciones y el NDVI de las dos comunidades seleccionadas (para cada mes se usó un promedio de los 100 pixeles para el período 2000/2016), incluyendo los dos meses anteriores y el mes considerado (i.e., calculado mediante una ventana móvil de tres meses) a fin de reducir la influencia de la variabilidad aleatoria interanual de las precipitaciones y el NDVI. Primero se realizó una comparación por mes para la precipitación y el NDVI, y luego por trimestre incluyendo el ONI. Para hacer el análisis comparativo categórico se compararon las medias de los valores por trimestre entre fases de ENSO utilizando la prueba de Tukey $(\alpha=0.05)$ para ONI (valor registrado por la NOAA para los trimestres considerados) y para precipitación y NDVI (integral de valores mensuales que componen los trimestres considerados). Esto permitió identificar y evaluar el retraso de la respuesta de la vegetación respecto de la ocurrencia de las precipitaciones y del ONI para las distintas fases del fenómeno ENSO.

Finalmente, se comparó la dinámica mensual del NDVI en dos años con eventos extremos La Niña y El Niño en las mismas dos comunidades de la Puna catamarqueña arriba mencionadas con otras dos comunidades afines de la Puna jujeña estudiadas por Maggi y Di Ferdinando (2012). Clasificadas como Puna seca y Puna semiárida, respectivamente según Morello et al. (2012). Las comunidades seleccionadas en la Puna jujeña fueron la estepa graminosa de Pennisetum chilense (E. Desv.) B. D. Jacks. ex R. E. Fr. y la estepa arbustiva de Parastrephia quadrangularis (Meyen) Cabrera (Morello et al. 2012). El estudio abarcó los meses desde octubre a abril, lapso que abarca la totalidad 
del período monzónico (González and Barros 1998; Liebmann and Marengo 2001; Rivera et al. 2009). Para hacer comparable el comportamiento de las diferentes comunidades frente al fenómeno ENSO, entre la Puna jujeña y la catamarqueña se escogieron las fases El Niño 2002/2003 y La Niña 2008/2009 (Barreda and Maggi 2018), correspondientes a eventos de sequía y precipitaciones intensas, respectivamente, en la Puna jujeña (según datos de las estaciones meteorológicas de Abra Pampa y La Quiaca [Maggi y Di Ferdinando 2012]). Para cada una de las cuatro comunidades, y para los dos períodos monzónicos comparados, se tomaron 100 valores mensuales de NDVI. Para cada fecha y comunidad se comparó el NDVI alcanzado en ambas fases mediante ANOVA (prueba de Tukey, $\alpha=0.05$ ), en los dos períodos monzónicos característicos seleccionados.

Además, se compararon los datos de NDVI obtenidos por los sensores MODIS y Landsat 5 TM para confirmar el comportamiento similar de la vegetación a lo largo del año, independientemente del sensor satelital utilizado. Para la extracción de datos de este último, teniendo en cuenta que la resolución es de $30 \mathrm{~m}$, se utilizó la mitad de la superficie de área de interés usada en MODIS. Se aplicó el Google Earth Engine. La Figura 2 muestra el flujo de trabajo.

\section{Resultados}

Se demostró que el NDVI es un buen indicador para diferenciar las comunidades de la Reserva. Aun cuando las ocho comunidades muestran bajos valores de NDVI, la mayoría de estos fueron estadísticamente diferentes (Tabla 1). Al estudiar los datos por trimestre, el índice

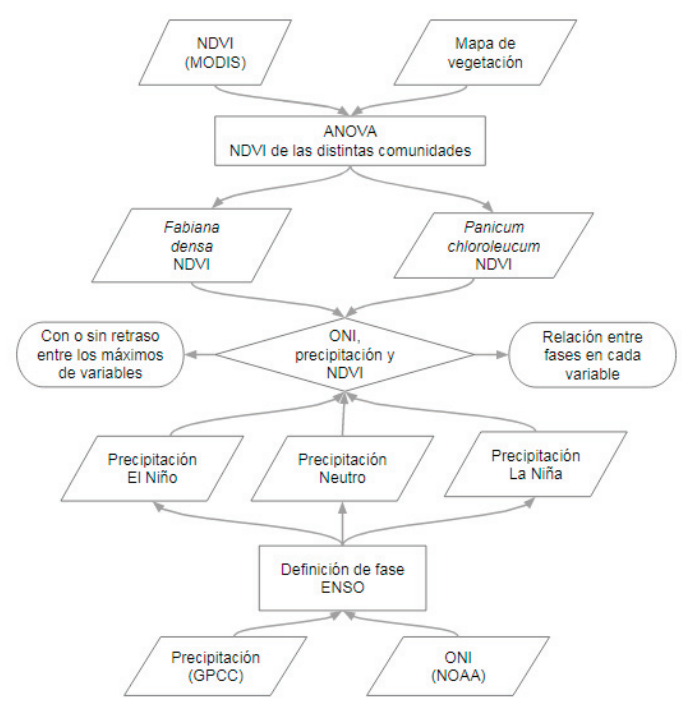

Figura 2. Flujo de trabajo.

Figure 2. Framework.

ONI muestra que las 3 fases del fenómeno ENSO se diferencian claramente en los dos primeros trimestres del monzón (septiembre a febrero) (Figura 3a). Las precipitaciones alcanzaron su valor máximo en el trimestre diciembre a febrero, siendo lo esperado para esta región (Figura 3b), sin mostrar diferencias significativas entre las fases. Sin embargo, cuando se analizan los valores mensuales, sólo en marzo las precipitaciones en la fase La Niña fueron significativamente mayores que en las otras dos fases (LSD de Fisher, $\alpha=0.10$ ) (Figura 4). El NDVI para Fabiana densa alcanza su pico máximo en el trimestre marzo-mayo, mostrando diferencias significativas entre las fases opuestas de El Niño y La Niña. Estas diferencias entre esas fases se mantienen para Panicum chloroleucum en el mismo trimestre. Sin embargo, en esta última comunidad los

Tabla 1. Resultados del ANOVA comparando los valores de NDVI del sensor MODIS registrados durante la serie 2000/2011 en todas las comunidades seleccionadas de Laguna Blanca. Medias con letras distintas muestran diferencias estadísticamente significativas entre comunidades (prueba de Tukey, $\alpha=5 \%$; $=100$ pixeles $\times 23$ imágenes $\times 12$ años; $\mathrm{N}=\mathrm{n} \times 8$ comunidades). La superficie se obtuvo del trabajo de Borgnia et al. (2006).

Table 1. Resulting ANOVA for all the selected communities in Laguna Blanca, based on NDVI values from MODIS sensor recorded during the period 2000/2011. Different letters show statistically significant difference among means of NDVI of the communities (Tukey test $\alpha=5 \%$ and $n=100$ pixels $\times 23$ images $\times 12$ years; $N=n \times 8$ communities). The area was obtained from Borgnia et al. (2006).

\begin{tabular}{lcccc}
\hline Comunidades & Sup. (ha) & NDVI media & NDVI desvío estándar & \\
\hline Estepa graminosa Panicum chloroleucum & 8182.5 & 0.116 & 0.001 & A \\
Estepa mixta Stipa sp. & 10738.8 & 0.101 & 0.001 & B \\
Estepa graminosa Stipa frigida y S. vaginata & 6582.2 & 0.087 & 0.001 & $\mathrm{C}$ \\
Estepa arbustiva Fabiana friesii, F. punensis & 11388.8 & 0.084 & 0.001 & $\mathrm{CD}$ \\
Estepa mixta Panicum chloroleucum & 10066.2 & 0.083 & 0.001 & $\mathrm{D}$ \\
Estepa arbustiva Acantholippia salsoloides & 5363.8 & 0.069 & 0.001 & $\mathrm{E}$ \\
Estepa arbustiva Fabiana densa & 15181.3 & 0.061 & 0.001 & $\mathrm{~F}$ \\
Estepa graminosa Festuca argentinensis & 11599.4 & 0.060 & 0.001 & $\mathrm{~F}$ \\
\hline
\end{tabular}



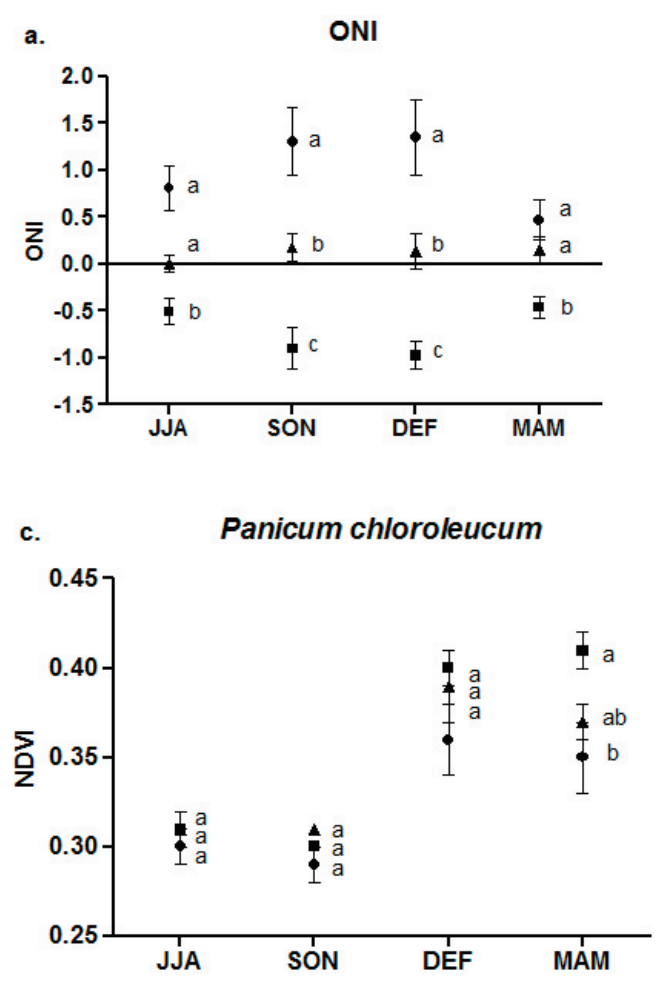

b.

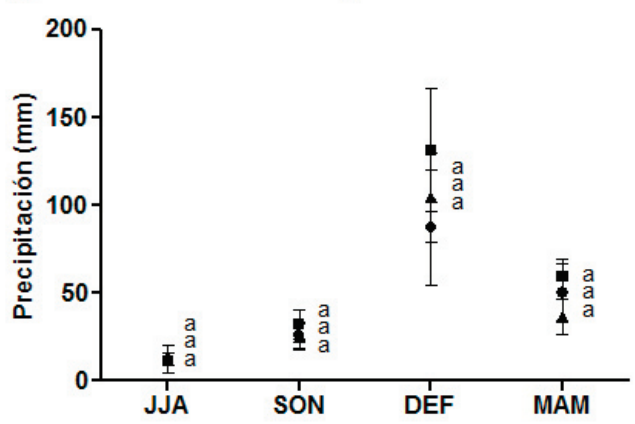

d.

Fabiana densa

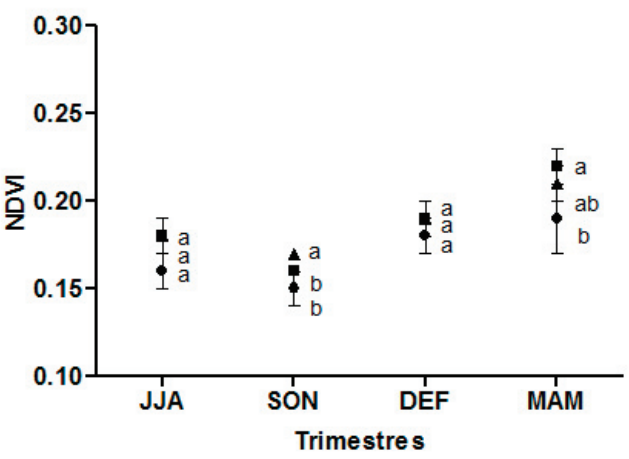

Figura 3. Valores trimestrales y su error estándar de las variables consideradas, en cada fase del fenómeno ENSO para el período 2000/2016. a) ONI; b) Promedio mensual de la precipitación estimada por el GPCC; c) NDVI estepa graminosa Panicum chloroleucum Griseb; d) NDVI estepa arbustiva Fabiana densa J. Rémy. Letras distintas indican diferencias estadísticamente significativas por trimestre entre las fases (prueba de Tukey, $\alpha=0.05$ ). Datos de NDVI obtenidos del sensor MODIS.

Figure 3. Quarterly values and its standard error of the variables considered, in each phase of the ENSO phenomenon for the period 2000/2016. a) ONI; b) Monthly average of the precipitation estimated by the GPCC; c) NDVI shrub steppe Fabiana densa J. Rémy; d) NDVI grass steppe Panicum chloroleucum Griseb. Different letters indicate statistically significant differences between phases per quarter (Tukey test, $\alpha=0.05$ ). NDVI values were obtained from MODIS sensor.

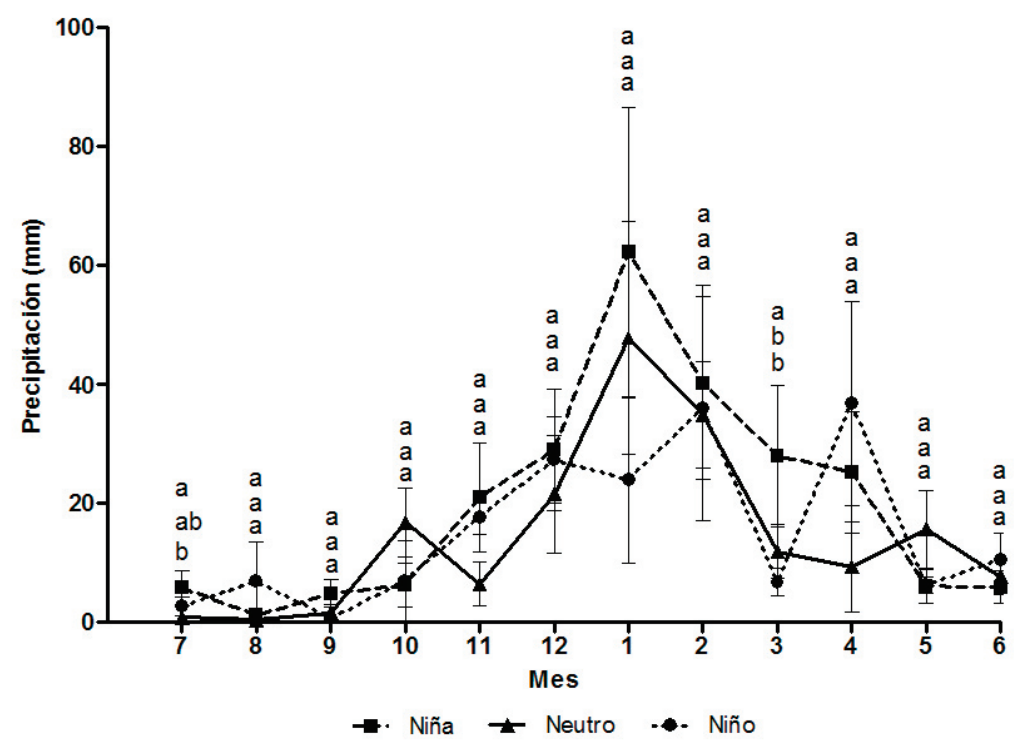

Figura 4. Precipitación mensual estimada y error estándar para la Reserva Laguna Blanca según las fases del fenómeno ENSO en el período 2000/2016. Datos obtenidos a partir del Global Precipitation Climatology Centre (GPCC). Letras distintas indican diferencias estadísticamente significativas por mes entre las fases (LSD de Fisher, $\alpha=0.10$ ).

Figure 4. Estimated monthly precipitation and standard error for Laguna Blanca according to the phases of the ENSO phenomenon in the period 2000/2016. Data obtained from the Global Precipitation Climatology Centre (GPCC). Different letters indicate statistically significant differences between phases per month (LSD de Fisher, $\alpha=0.10$ ). 
valores de NDVI más elevados se sostienen entre diciembre y mayo, sugiriendo una rápida respuesta al período de precipitaciones más abundantes del monzón (Figura 3b, c y d).

El análisis temporal más detallado (Figura 5) confirma que el pico de NDVI mostró un retraso respecto al de precipitación en ambas estepas. En efecto, la precipitación alcanzó sus máximos valores en febrero, mientras que el NDVI lo alcanzó entre marzo y abril. Por su parte, la estepa de gramíneas (Figura 5a) mostró valores de NDVI mucho más elevados que la estepa arbustiva debido a sus diferentes coberturas basales (Figura 5b).
Tanto los valores de precipitación como los de NDVI, durante la fase La Niña superaron la fase El Niño en verano y otoño en ambas comunidades.

La comparación entre las estepas graminosas y arbustivas de la Puna catamarqueña y jujeña muestra que las cuatro comunidades escogidas presentan los valores más elevados de NDVI durante el período estival. Dichos valores, además fueron significativamente mayores durante La Niña 2008/2009 respecto de El Niño 2002/2003 (Figura 6). Las comunidades de la Puna jujeña presentaron registros más elevados de NDVI que los de la Puna

a.

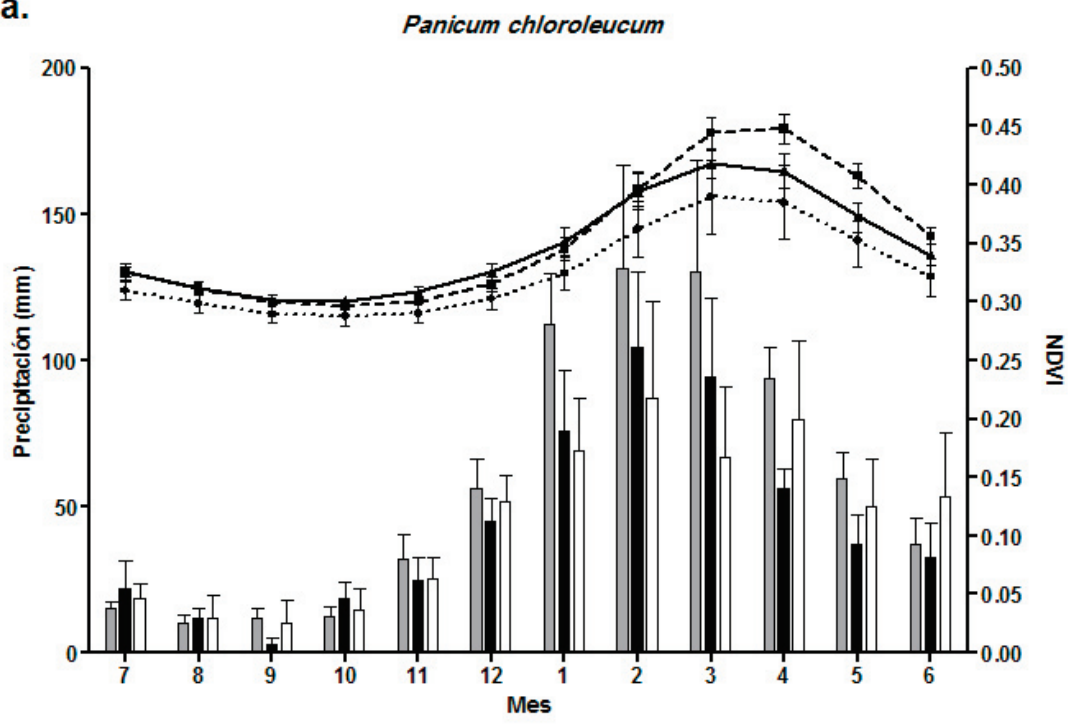

Figura 5. Integral de tres meses del promedio mensual de la precipitación estimada por el GPCC y el mismo período de integración del promedio mensual de NDVI (sensor MODIS), para las fases del fenómeno ENSO en la serie $2000 / 2016$. a) Comunidad graminosa Panicum chloroleucum Griseb; b) Comunidad arbustiva Fabiana densa J. Rémy. Las barras de error representan el error estándar del

b.

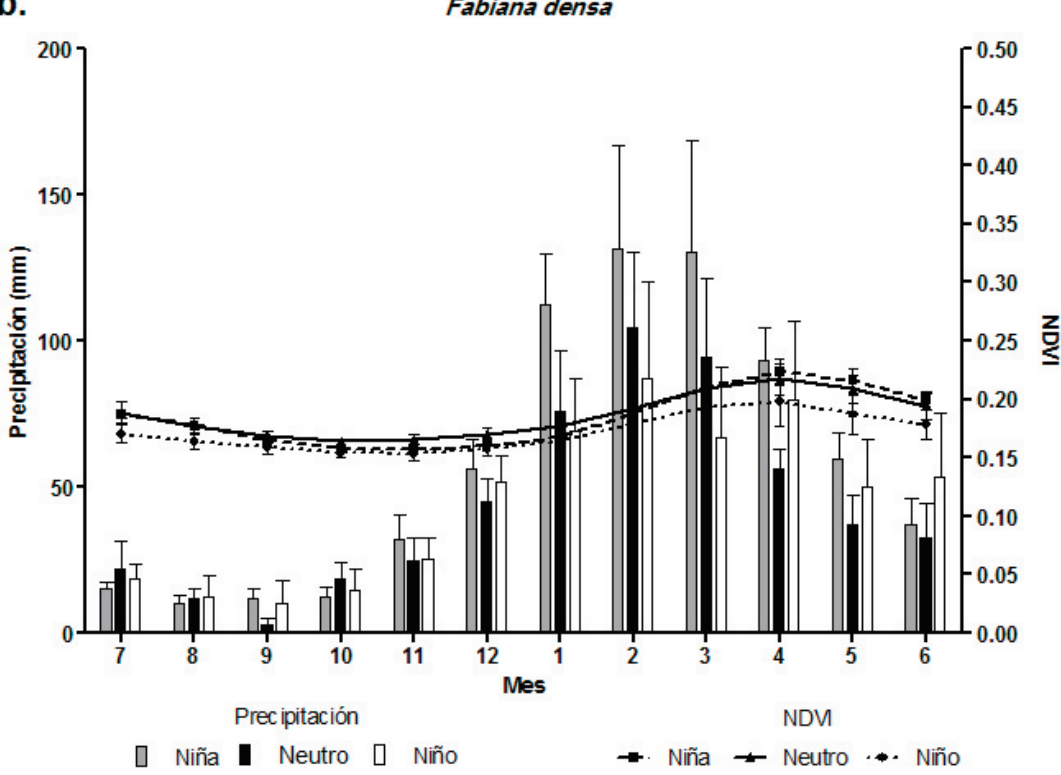

NDVI y la mitad del error estándar de la precipitación.

Figure 5. Integral of three months of the monthly average of the precipitation estimated by the GPCC and the same integration period of the monthly average of NDVI (MODIS sensor), for the phases of the ENSO phenomenon in the $2000 / 2016$ period. a) Grass community of Panicum chloroleucum Griseb; b) Shrub community of Fabiana densa J. Rémy. Error bars represent the standard error of NDVI and half of the standard error of precipitation. 
a.

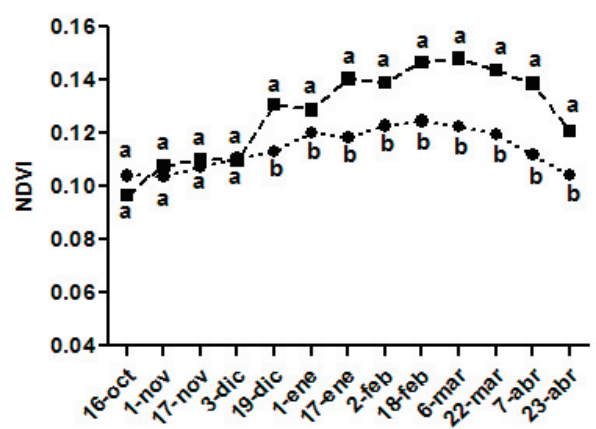

c.

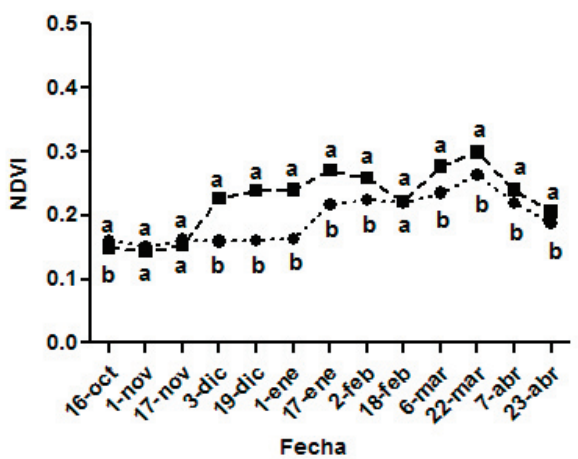

b.

Fabiana densa

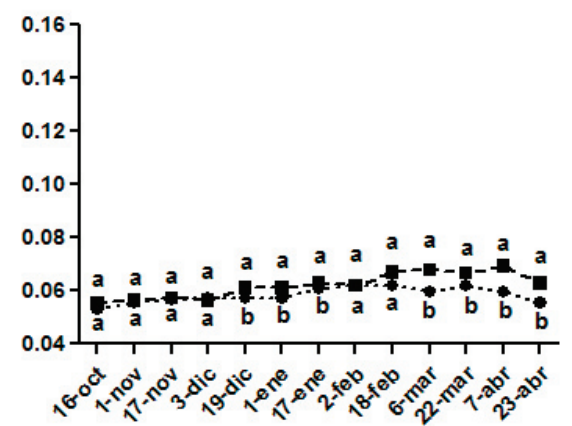

d.

Parastrephia quadrangularis

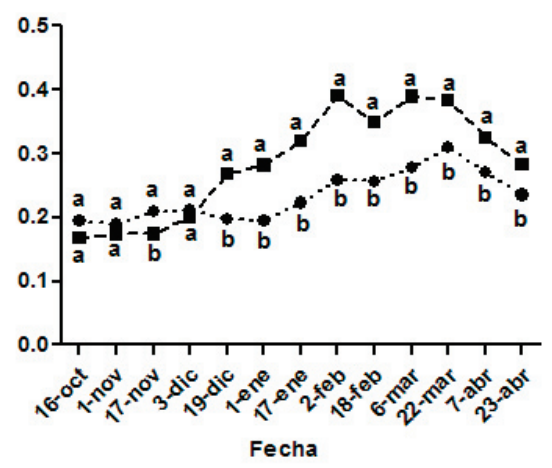

El Niño 2002-2003

Figura 6. Valores de NDVI del sensor MODIS durante La Niña 2008/2009 y El Niño 2002/2003 para las comunidades: a) Estepa graminosa Panicum chloroleucum Griseb; b) Estepa arbustiva Fabiana densa J. Rémy; c) Estepa graminosa Pennisetum chilense (E. Desv.) B. D. Jacks. ex R. E. Fr.; d) Estepa arbustiva Parastrephia quadrangularis (Meyen) Cabrera. a y $\mathrm{b}=$ Comunidades de la Puna catamarqueña; $\mathrm{c}$ y d=Comunidades de la Puna jujeña. Letras distintas muestran diferencias estadísticamente significativas entre fases dentro de cada fecha (Prueba de Tukey, $\alpha=0.05$ ).

Figure 6. NDVI values from MODIS sensor during La Niña 2008/2009 and El Niño 2002/2003 for the selected communities: a) Grass steppe Panicum chloroleucum Griseb; b) Shrub steppe Fabiana densa J. Rémy; c) Grass steppe Pennisetum chilense (E. Desv.) B. D. Jacks. ex R. E. Fr.; d) Shrub steppe Parastrephia quadrangularis (Meyen) Cabrera. a and $b=$ Communities from the dry Puna in Catamarca; $c$ and $d=$ Communities from the semiarid Puna in Jujuy. Different letters indicate statistically significant differences in each date (Tukey Test, $\alpha=0.05$ ).
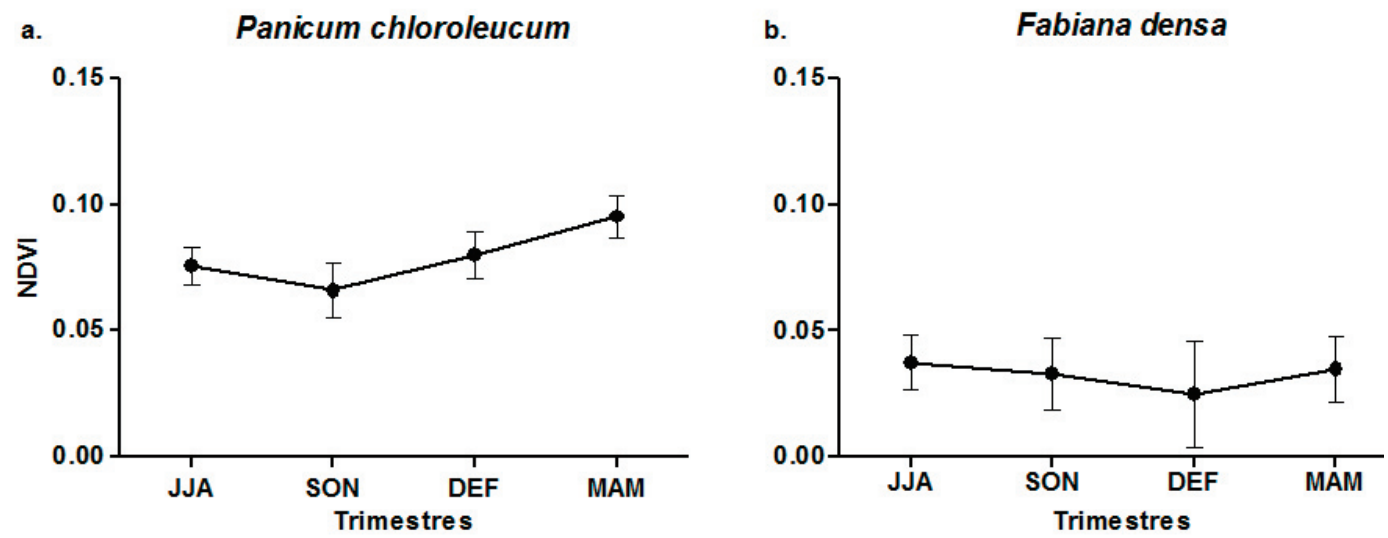

Figura 7. Media (X) y desvío estándar (DE) del NDVI del satélite Landsat 5 TM de cada trimestre en el período 20002012 para las comunidades: a) Estepa graminosa Panicum chloroleucum Griseb, y b) Estepa arbustiva Fabiana densa J. Rémy en la Puna Catamarqueña.

Figure 7. Mean (X) and standard deviation (SD) of NDVI of the Landsat 5 TM satellite of each quarter in the period 2000-2012 for the communities: a) Grass steppe Panicum chloroleucum Griseb, and b) Shrub steppe Fabiana densa J. Rémy in the dry Puna in Catamarca. 
seca catamarqueña. El NDVI muestra las mayores diferencias entre los dos eventos extremos analizados hacia final del período monzónico.

Los valores más elevados de NDVI para Panicum chloroleucum se producen en el trimestre marzo-mayo, medidos tanto en imágenes Landsat 5 TM como en MODIS (Figuras 3 y 7). Las diferencias entre ambos sensores se deben a que en Landsat se usó la mitad de la superficie de área de interés y distintas fechas de muestreo. La comunidad de Fabiana densa muestra valores de NDVI mucho menores que la de Panicum chloroleucum, para ambos sensores. La escasa cantidad de imágenes Landsat no permitieron hacer una comparación entre fases. Los valores de Fabiana densa no muestran un comportamiento razonable y deberán hacerse más estudios; es posible que al aumentar la resolución espacial en una comunidad con baja cobertura se modifiquen los resultados observados con MODIS.

\section{Discusión}

De manera similar a lo obtenido por Rojo et al. (2019) en la Puna semiárida argentina, el NDVI permitió diferenciar la mayoría de las unidades de vegetación en nuestra área de estudio ubicada en la Puna seca. Esta investigación demuestra que, en general en la Puna seca de Catamarca, las precipitaciones son más elevadas en los años correspondientes a la fase La Niña del fenómeno ENSO que en los correspondientes a las otras fases y eso se refleja en diferencias en el NDVI. Si bien las precipitaciones mensuales en cualquiera de las fases muestran una marcada variabilidad interanual que dificulta la detección de diferencias estadísticamente significativas, durante el mes de marzo los años La Niña se mostraron significativamente más lluviosos que los años Neutro o El Niño. Por otra parte, en otras regiones de la Puna también se pudo distinguirentrelas fases del fenómeno ENSOen términos de precipitación acumulada durante el período monzónico, especialmente en la fase El Niño, usando datos provenientes de muchos años de registro de estaciones meteorológicas puntuales (Vuille and Bradley 2000; Garreaud and Aceituno 2007; Bennett et al. 2016; Barrera and Maggi 2018). Las disparidades halladas en la cantidad de precipitaciones se reflejaron en diferencias en el NDVI, índice espectral ligado tanto a la PPNA como a la cobertura vegetal. El NDVI fue mayor en los años La Niña en las dos comunidades de la estepa de la Puna catamarqueña aquí analizadas (Figuras 3 y 5), aunque tal diferencia fue más significativa en la estepa graminosa de Panicum chloroleucum que en la arbustiva de Fabiana densa. Ese patrón se repitió y se manifiesta de manera significativa a partir de noviembre al comparar la incidencia de años extremos La Niña y El Niño en esas mismas dos comunidades y en dos comunidades afines de la Puna jujeña, más húmedas. Ambas comunidades escogidas por su cobertura vegetal, arbustivas y graminosas siguen un patrón estacional de crecimiento del tipo monzónico, similar a los encontrados en la Puna jujeña. Sin embargo, pudimos comprobar que los valores de NDVI entre comunidades de tipos fisonómicos similares son de mucha menor magnitud en la Puna catamarqueña a los encontrados en Jujuy, evidenciando menores precipitaciones durante la estación lluviosa y una menor cobertura vegetal presente en la Puna seca. La diferencia en los valores de NDVI entre fases es máxima en la estepa arbustiva jujeña de Parastrephia quadrangularis, especie adaptada al ascenso de la capa freática (Ruthsatz and Movia 1975; Paoli et al. 2002; Matteucci et al. 2017) y mínima en la estepa arbustiva catamarqueña de Fabiana densa; justamente, las estepas de mayor y menor cobertura vegetal respectivamente (Figuras $6 \mathrm{~d}$ vs. 6b). Estos resultados son coincidentes con los obtenidos por Anyamba et al. y Anyamba y Tucker en los años 2002 y 2005, quienes demostraron que los cambios de los patrones de precipitación inducidos por las condiciones de El Niño y La Niña tuvieron efectos significativos en la producción de biomasa en tierras áridas y semiáridas de África. Por lo tanto, los años La Niña podrían jugar un rol muy importante al determinar condiciones más favorables para el aumento de cobertura vegetal en la Puna.

Otro hallazgo importante de nuestro trabajo fue la identificación de un retraso en la respuesta de las precipitaciones de un trimestre respecto a la definición de la fase del ENSO en el Océano Pacífico (reflejada en el índice ONI) y, a su vez, la respuesta del NDVI muestra un retraso de un trimestre o menos respecto a la ocurrencia de la máxima precipitación mensual en todas las fases del ENSO (Figura 3). En un análisis más detallado entre la precipitación y el NDVI, este retraso se manifiesta entre uno a dos meses tanto en las estepas graminosas de Panicum chloroleucum como en las arbustivas de Fabiana densa (Figuras 5). En las comunidades de Matorrales 
de Parastrephia quadrangularis y de Pastizales de Pennisetum chilense de la Puna jujeña se observó un retraso similar del pico de NDVI respecto al de las precipitaciones, aunque de mayor magnitud y con valores de NDVI 50\% más elevados, ya que las precipitaciones son mayores (Maggi and Di Ferdinando 2012) (Figura 6). Estos resultados acompañan a las precipitaciones disminuyendo de norte a sur y de este a oeste en toda esta ecorregión (Bennett et al. 2016). Este retraso en la respuesta de la vegetación a los pulsos de humedad edáfica que siguen a la ocurrencia de las lluvias también fue mostrado en ambientes similares de Israel (Schmidt and Karnieli 2000), en África al oeste (Philippon et al. 2005) y al sur (Wiegand et al. 2008) y en la Argentina, para la estepa patagónica (Fabricante et al. 2009).

El conocimiento de la dinámica de la vegetación es relevante para comprender los procesos de tierras áridas (Thomas et al. 2011), en particular aquellos asociados con la degradación de la tierra (Tchilinguirian and Olivera 2012). Aun con bajos valores de NDVI, estos resultados explicarían la existencia de la teleconexión del fenómeno ENSO y las precipitaciones en la Puna seca catamarqueña, con su incidencia en la producción forrajera de las estepas y las variaciones en la cobertura del suelo, todavía no demostrada por otros autores.

Además, en términos del diseño de estrategias de control de la desertificación, el monitoreo del índice ONI, al presentar las diferencias en el trimestre septiembre-noviembre, permitiría anticipar en un trimestre la ocurrencia de lluvias o sequías extraordinarias. En uno a dos trimestres permitiría anticipar la ocurrencia de oportunidades para aumentar la cobertura en determinados sitios estratégicos en los años La Niña o, por el contrario, establecer clausuras y reducir al mínimo la presión de pastoreo en sitios muy frágiles durante los años El Niño, evitando mortandad de animales y sobrepastoreos que disparan los procesos de erosión y la pérdida de servicios ecosistémicos. Estas alarmas tempranas también fueron descriptas en Asia y África en especial en los eventos fuertes (Aralova et al. 2016; Anyamba et al. 2018). Del mismo modo que este último autor, se podrían desarrollar ajustes para poder predecir con exactitud qué cantidad de meses de retraso ocurren entre las variables climáticas y el NDVI para las diversas regiones de la Puna definidas por Cabrera (1976) y Morello et al. (2012).

\section{CONCLUSIONES}

Este trabajo permitió caracterizar la dinámica de la cobertura vegetal y sus relaciones con las oscilaciones de las precipitaciones intra e interanuales en una región que posee pocos datos e información. El NDVI obtenido a partir del sensor MODIS permitió separar las distintas comunidades vegetales identificadas en la Reserva de Biósfera Laguna Blanca.

Se probó parcialmente la relación entre las fases del ENSO y las precipitaciones y su efecto sobre la vegetación de estepas estimada a través del NDVI en la reserva. Para la comunidad de Panicum chloroleucum, al comparar mensualmente los valores medios de NDVI de todos los monzones incluidos en el período 2000-2016, sólo se pudo probar diferencias significativas durante los meses de abril y mayo entre los años con las fases La Niña y El Niño. Ello evidencia algún grado de teleconexión climática del fenómeno ENSO al final de la estación de lluvias que afecta las precipitaciones y el NDVI en esta comunidad; sugiriendo que los efectos de las alternancias de las fases del ENSO en el NDVI, producirían cambios en la PPNA y la cobertura del suelo.

Se demostró un retraso de un trimestre de la máxima precipitación y un retraso de uno a dos trimestres del pico del NDVI respecto del índice ONI en la Puna seca argentina. Estos resultados aportan mucha información que contribuye a la comprensión de los efectos del fenómeno ENSO en la cobertura vegetal de la Puna de Catamarca y al acercamiento de un plan integral de manejo para evitar el sobrepastoreo en la zona. No obstante, se recomienda profundizar las investigaciones en la Puna seca con series más largas que permitan confirmar las relaciones existentes entre el fenómeno ENSO, las precipitaciones y el NDVI halladas aquí.

Agradecimientos. Los autores agradecen sugerencias y aportes realizados por el Dr. R. Golluscio y el Dr. H. F. del Valle. Al Sr. M. Bosio en el procesamiento de datos con Google Earth Engine. Este trabajo de investigación fue financiado por el proyecto UBACyT20020130200156BA, UBACYT 20142017 GEF. 


\section{REFERENCIAS}

Agosta, E. A., and R. H. Compagnucci. 2008. The 1976/77 austral summer climate transition effects on the atmospheric circulation and climate in southern South America. Journal of Climate 21(17):4365-4383. https://doi.org/10.1175/ 2008JCLI2137.1.

Anyamba, A., E. Glennie, and J. Small. 2018. Teleconnections and Interannual Transitions as Observed in African Vegetation: 2015-2017. Remote Sensing 10(7):1038. https:/ / doi.org/10.3390/rs10071038.

Anyamba, A., C. J. Tucker, and R. Mahoney. 2002. From El Niño to La Niña: Vegetation Response Patterns over East and Southern Africa during the 1997-2000 Period. Journal of Climate 15:3096-3103. https://doi.org/10.1175/15200442(2002)015\%3C3096:FENOTL\%3E2.0.CO;2.

Anyamba, A., and C. J. Tucker. 2005. Analysis of Sahelian vegetation dynamics using NOAA-AVHRR NDVI data from 1981-2003. Journal of Arid Environments 63:596-614. https://doi.org/10.1016/j.jaridenv.2005.03.007.

Aralova, D., K. Toderich, B. Jarihani, D. Gafurov, and L. Gismatulina. 2016. Monitoring of vegetation condition using the NDVI/ENSO anomalies in Central Asia and their relationships with ONI (very strong) phases. In Earth Resources and Environmental Remote Sensing/GIS Applications VII (Vol. 10005, Pp. 1000512). International Society for Optics and Photonics. https://doi.org/10.1117/12.2242164.

Baldassini, P., J. N. Volante, L. M. Califano, and J. M. Paruelo. 2012. Caracterización regional de la estructura y de la productividad de la vegetación de la puna mediante el uso de imágenes MODIS. Ecología Austral 22:22-32.

Barrera, D. F., and A. E. Maggi. 2018. Variabilidad de la precipitación en el altiplano argentino. Incidencia de la transición climática 1976/1977 y del Fenómeno El Niño-Oscilación del Sur en el Noroeste Argentino. Revista Meteorológica 43(1):41-71

Barros, V. 2004. Cambio climático global. Libros del zorzal.

Barros, V. R., and I. Camilloni. 2016. La Argentina y el cambio climático: de la física a la política. Eudeba. Pp. 286.

Bennett, M., M. New, J. Marino, and C. Sillero-Zubiri. 2016. Climate complexity in the Central Andes: a study case on empirically-based local variations in the Dry Puna. Journal of Arid Environments 128:40-49. https://doi.org/ 10.1016/j.jaridenv.2016.01.004.

Borgnia, M., A. E. Maggi, M. Arriaga, B. Aued, B. L. Vilá, and M. H. Cassini. 2006. Caracterización de la vegetación en la Reserva de Biósfera Laguna Blanca (Catamarca, Argentina). Ecología Austral 16:29-45.

Cabrera, A. L. 1976. Regiones fitogeográficas argentinas. En W. F. Kugler (ed.). Enciclopedia argentina de agricultura y jardinería 2(1):1-85. Ed. Acme, Buenos Aires, Argentina.

Carlson, T. N., and D. A. Ripley. 1997. On the Relation between NDVI, Fractional Vegetation Cover, and Leaf Area Index. Remote Sensing of Environment 62:241-252. https:/ /doi.org/10.1016/S0034-4257(97)00104-1.

Chen, M., W. J. Parton, S. J. Del Grosso, M. D. Hartman, K. A. Day, C. J. Tucker, J. D. Derner, A. Knapp, W. K. Smith, D. S. Ojima, and W. Gao. 2017. The signature of sea surface temperature anomalies on the dynamics of semiarid grassland productivity. Ecosphere 8(12):e02069. https://doi.org/10.1002/ecs2.2069.

Espoz-Alsina, C., S. M. Navone, and A. E. Maggi. 2002. Development of a desertification assessment method using a geographic information system: a case study in northwestern Argentina. Management Information Systems 2002: Incorporating Gis and Remote Sensing (Management Information Systems, V. 4)-US-ISBN:1853129070 (Hard cover book) Publisher: Wit Pr/Computational Mechanics Published 2002/02BakeryTaylor. Pp. 448.

Fabricante, I., M. Oesterheld, and J. M. Paruelo. 2009. Annual and seasonal variation of NDVI explained by current and previous precipitation across Northern Patagonia. Journal of Arid Environments 73(8):745-753. https:/ / doi.org/ 10.1016/j.jaridenv.2009.02.006.

First, P. J. 2019. Global Warming of $1.5^{\circ} \mathrm{C}$. An IPCC Special Report on the Impacts of Global Warming of $1.5 \mathrm{C}$ Above Pre-Industrial Levels and Related Global Greenhouse Gas Emission Pathways, in the Context of Strengthening the Global Response to the Threat of Climate Change. Sustainable Development, and Efforts to Eradicate Poverty. URL: www.ipcc.ch/sr15/.

Gaitán, J. J., D. Bran, G. Oliva, G. Ciari, V. Nakamatsu, J. Salomone, and D. Celdrán. 2013. Evaluating the performance of multiple remote sensing indices to predict the spatial variability of ecosystem structure and functioning in Patagonian steppes. Ecological Indicators 34:181-191. https://doi.org/10.1016/j.ecolind.2013.05.007.

Gao, X., A. R. Huete, and K. Didan. 2003. Multisensor Comparisons and Validation of MODIS Vegetation Indices at the Semiarid Jornada Experimental Range. IEEE Transactions Geoscience and Remote Sensing 41(10):2368-2381. https: //doi.org/10.1109/TGRS.2003.813840.

Garreaud, R. D., and P. Aceituno. 2007. Atmospheric circulation over South America: mean features and variability. Pp. 45-66 in T. Veblen, K. Young and A. Orme (eds.). The Physical Geography of South America. Oxford University Press, Oxford.

González, M., and V. Barros. 1998. The relation between tropical convection in South América and the end of the dry period in subtropical Argentina. International Journal of Climatology 18:1669-1685. https://doi.org/10.1002/ (SICI)1097-0088(199812)18:15\%3C1669::AID-JOC340\%3E3.0.CO;2-1.

IPCC. 2014. Climate Change 2013-The Physical Science Basis: Working Group I Contribution to the Fifth Assessment Report of the Intergovernmental Panel on Climate Change. Cambridge University Press, UK.

Liebmann, B., and J. A. Marengo. 2001. Interannual Variability of the Rainy Season and Rainfall in the Brazilian Amazon Basin. Journal of Climate 14:4308-4318. https://doi.org/10.1175/1520-0442(2001)014\%3C4308: IVOTRS\%3E2.0.CO;2.

Maccagno, P. 2004. Indicadores socioeconómicos de desertificación en la región de la puna. En Teledetección Aplicada 
a la Problemática ambiental Argentina. Centro de Investigación y Aplicación a la Teledetección de la FAUBA. Pp. 31-40. Editorial EFA.

Maggi, A., and M. Di Ferdinando. 2012. Efecto del fenómeno ENOS en la respuesta del NDVI de la estepa arbustiva de Parastrephia sp. de la laguna de Pozuelos usando imágenes MODIS. Congreso Argentino de Teledetección.

Maggi, A., S. M. Navone, and F. A. Kindgard. 2010. Monitoreo de los cambios en el comportamiento de algunas lagunas debido a la Oscilación Climática utilizando imágenes satelitales en la Puna Jujeña. Edición Especial de la Revista Selper 2:34-45.

Maggi, A., and K. Ponieman. 2018. Changes in vegetal cover, precipitations and land degradation in Puna region, Argentina. Modern Environmental Science and Engineering 4(7):638-643.

Mantua, N. J., and S. R. Hare. 2002. The Pacific decadal oscillation. Journal of Oceanography 58(1):35-44. https:// doi.org/10.1023/A:1015820616384.

Matteucci, S. D., A. F. Rodríguez, and M. E. Silva. 2017. La vegetación de la Argentina. Universidad de Buenos Aires. Facultad de Arquitectura y Urbanismo. Grupo de Ecología del Paisaje y Medio Ambiente. Fronteras 15(6):4-29

Maturana, J., M. Bello, and M. Manley. 1997. Antecedentes históricos y descripción del fenómeno El Niño, Oscilación del Sur. Pp. 13-27 en S. Avaria, J. Carrasco, J. Rutllant and E. Yáñez (eds.). 2004. El Niño-La Niña 1997-2000. Sus Efectos en Chile. CONA, Chile, Valparaíso.

Minetti, J. L., and W. M. Vargas. 1997. Trends and jumps in the annual precipitation in South America, south of the 15o S. Atmósfera 11:205-221.

Minvielle, M., and R. D. Garreaud. 2011. Projecting Rainfall Changes over the South American Altiplano. Journal of Climate 9:4577-4583. https://doi.org/10.1175/JCLI-D-11-00051.1.

Morello, J., S. D. Matteucci, A. Rodríguez, and M. Silva. 2012. Ecorregiones y complejos ecosistémicos de argentina. Buenos Aires: Orientación Gráfica Editora.

Movia, C., and S. M. Navone. 1993. Imágenes Landsat TM: Una herramienta para evaluar el deterioro de los pastizales en la Puna Argentina. Investigación Agraria, Producción y Protección Vegetales (Argentina) 9:30-35.

Paruelo, J. M., and W. K. Lauenroth. 1998. Interannual variability of NDVI and its relationship to climate for North American shrublands and grassland. Journal of Biogeography 25:721-733. https://doi.org/10.1046/j.13652699.1998.2540721.x.

Paoli, H., A. R. Bianchi, C. E. Yáñez, J. N, Volante, D. R. Fernández, M. C. Mattalía, and Y. E. Noé. 2002. Recursos Hídricos de la Puna, valles y Bolsones áridos del Noroeste Argentino. Convenio INTA EEA Salta-CIED.

Philippon, N., E. Mougin, L. Jarlan, and P. L. Frison. 2005. Analysis of the linkages between rainfall and land surface conditions in the West African monsoon through CMAP, ERS-WSC, and NOAA-AVHRR data. Journal of Geophysical Research Atmospheres 110:D24115. https://doi.org/10.1029/2005JD006394.

Ponieman, K., A. Maggi, and P. Baldassini. 2018. Ecuaciones para la estimación de la cobertura vegetal en estepas de la puna a partir de IVN de MODIS. VIII Congreso Nacional de Manejo de Pastizales Naturales y el IV Congreso del Mercosur de Manejo de Pastizales Naturales.

Rivera, J. A., N. Herrera, C. N. Gulizia, N. B. Montroull, and P. Spennemann. 2009. Identificación del comienzo de la estación lluviosa en la región subtropical de Argentina. Actas de la XXIV Reunión Científica de la AAGG, 1: 61-67. Asociación Argentina de Geofísicos y Geodestas, Buenos Aires. ISBN 978-987-25291-1-6.

Rojo, V., Y. Arzamendia, C. Pérez, J. Baldo, and B. L. Vilá. 2019. Spatial and temporal variation of the vegetation of the semiarid Puna in a pastoral system in the Pozuelos Biosphere Reserve. Environmental Monitoring and Assessment 191(10):635. https://doi.org/10.1007/s10661-019-7803-7.

Ruthsatz, B., and C. P. Movia. 1975. Relevamiento de las estepas altoandinas del noreste de la provincia de Jujuy, República Argentina. Fundación Educación, Ciencia, Cultura. Buenos Aires.

Schmidt, H., and A. Karnieli. 2000. Remote sensing of the seasonal variability of vegetation in a semi-arid environment. Journal of Arid Environments 45(1):43-59. https:/ / doi.org/10.1006/jare.1999.0607.

Seiler, C., R. W. Hutjes, and P. Kabat. 2013. Climate variability and trends in Bolivia. Journal of Applied Meteorology and Climatology 52(1):130-146. https://doi.org/10.1175/JAMC-D-12-0105.1.

Sellers, P. J., J. A. Berry, G. J. Collatz, C. B. Field, and F. G. Hall. 1992. Canopy reflectance, photosynthesis, and transpiration. III. A reanalysis using improved leaf models and a new canopy integration scheme. Remote Sensing of Environment 42(3):187-216. https: / /doi.org/10.1016/0034-4257(92)90102-P.

Tchilinguirian, P., and D. E. Olivera. 2012. Degradación y formación de vegas puneñas (900-150 años AP), Puna Austral (26 S) ¿Respuesta del paisaje al clima o al hombre. Acta Geológica 24:41-61.

Thibeault, J., A. Seth, and G. Wang. 2011. Mechanisms of summertime precipitation variability in the Bolivian Altiplano: present and future. International Journal of Climatology 32(13):2033-2041. https://doi.org/10.1002/joc.2424.

Thomas, E., D. Douterlungne, I. Vandebroek, F. Heens, P. Goetghebeur, and P. Van Damme. 2011. Human impact on wild firewood species in the Rural Andes community of Apillapampa, Bolivia. Environmental Monitoring and Assessment 178:333-347. https://doi.org/10.1007/s10661-010-1693-z.

Trauth, M. H., R. A. Alonso, K. R. Haselton, R. L. Hermanns, and M. R. Strecker. 2000. Climate change and mass movements in the NW Argentine Andes. Earth and Planetary Science Letters 179:243-256. https:/ / doi.org/10.1016/ S0012-821X(00)00127-8.

Tsonis, A., A. G. Hunt, and J. B. Elsner. 2003. On the relation between ENOS and global climate change. Meteorology and Atmospherics Physics 000:1-14.

UN (United Nations). 1994. United Nations Convention to Combat Desertification in Countries Experiencing Serious 
Drought and/or Desertification, Particularly in Africa. Document A/AC. 241/27, 12. 09. 1994 with Annexes, United Nations: New York, NY.

Vuille, M., and R. S. Bradley. 2000. Mean annual temperature trends and their vertical structure in the tropical Andes. Geophys Res Lett 27:3885-3888. https://doi.org/10.1029/2000GL011871.

Vuille, M., R. S. Bradley, M. Werner, and F. Keimig. 2003. 20th Century Climate Change in the Tropical Andes: Observations and Model Results. In H. F. Díaz (ed.). Climate Variability and Change in High Elevation Regions: Past, Present y Future. Advances in Global Change Research. Vol 15. Springer, Dordrecht. https:/ / doi.org/10.1007/97894-015-1252-7_5.

Wiegand, T., H. A. Snyman, K. Kellner, and J. M. Paruelo. 2008. Do Grasslands Have a Memory: Modeling Phytomass Production of a Semiarid South African Grassland. Ecosystems 7:243-258. https://doi.org/10.1007/s10021-0030235-8.

Worcel, L., and A. E. Maggi. 2016. Impacto del fenómeno ENOS en comunidades vegetales de la puna salteña. Puerto Iguazú XVII Simposio Internacional SELPER. 